ization of cervical cancer cases to high-volume centers provides an increase in fellows' exposure to radical procedures. LACC-trial publication did not reduce the possibility for a fellow to perform radical hysterectomies, but it may have affected the opportunity of performing minimally-invasive radical hysterectomy.

\section{COVID-19 VACCINATION PRIOR TO GYNAECOLOGICAL ONCOLOGY SURGERY : VACCINE COMPLIANCE AND PERI-OPERATIVE OUTCOMES IN A TERTIARY CANCER INSTITUTE IN INDIA}

${ }^{1} \mathrm{R}$ Modi ${ }^{*},{ }^{2} \mathrm{G}$ Pandey, ${ }^{3} \mathrm{~S}$ Chauhan, ${ }^{4} \mathrm{~S}$ Saini, ${ }^{4} \mathrm{M}$ Gupta, ${ }^{4} \mathrm{~S}$ Verma. ${ }^{1}$ Cancer Research Institute, Gynaecological Oncology services, India; ${ }^{2}$ Himalayan Institute of Medical Sciences, Surgery; ${ }^{3}$ Himalayan Institute of Medical Sciences; ${ }^{4}$ Cancer Research Institute

\subsection{6/ijgc-2021-ESGO.299}

Introduction/Background* India experienced a deadly second wave of COVID-19 pandemic starting mid-February 2021 with test positivity rate of 25-45\% suggesting high community transmission. Indian COVID-19 vaccination program for 60 years + and above 45 years with co-morbidities began on 1st March 2021. As per COVIDsurg collaborative data, between $0.6 \%$ and $1.6 \%$ of patients develop COVID-19 infection after elective surgery. Even after use of mitigation measures like pre-surgery RT/PCR and COVID free surgical pathways, COVID-19 is a significant nosocomial infection with 4- and 8-fold increased risk of death in the 30 days following surgery. Our aim was to study vaccine compliance in patients counselled to be vaccinated before surgery, pre-surgery RT/PCR positivity rate, 30-day post-operative SARS Cov-2 rate and peri-operative outcomes.

Methodology In this prospective observational study, patients waitlisted for major gynaecological cancer surgeries who were also eligible for COVID-19 vaccination were enrolled. Patients were counselled to get atleast one dose vaccinated 2 weeks before elective surgery. In cases of neo-adjuvant chemotherapy, vaccination was advised atleast 2 weeks after the last dose of chemotherapy. Patients vaccinated with atleast 1 dose - 2 weeks prior to surgery or those with both doses vaccinated atleast a week prior to surgery were eligible for study. Mitigation measures of negative pre-surgery RT/PCR (within 24 hours prior to surgery) and COVID free surgical pathway were used.

Abstract 313 Table 1 Vaccination, RT/PCR positively and perioperative outcome details

\begin{tabular}{|c|c|c|}
\hline \multicolumn{3}{|c|}{ Overall Cohort -53 patients } \\
\hline Median age (Range) & 51 years $(45-70$ & \\
\hline \multicolumn{3}{|c|}{ Vaccination Status } \\
\hline Unvaccinated & \multirow{2}{*}{\multicolumn{2}{|c|}{$\begin{array}{l}19 \\
25\end{array}$}} \\
\hline 1 dose vaccinated & & \\
\hline 2 dose vaccinated & \multicolumn{2}{|l|}{9} \\
\hline Vaccine compliance & $64 \%$ & \\
\hline \multicolumn{3}{|c|}{ Pre-surgery RT/PCR positivity rate } \\
\hline Unvaccinated & $10 / 19(52.6 \%)$ & \multirow[b]{2}{*}{$p=0.0001$} \\
\hline $\begin{array}{l}\text { Vaccinated } \\
\text { 1-dose } \\
\text { 2-dose }\end{array}$ & $\begin{array}{l}2 / 34(5.8 \%) \\
2 \\
0\end{array}$ & \\
\hline \multicolumn{3}{|c|}{ Severe COVID-19 infection requiring hospitalisation } \\
\hline Unvaccinated & \multicolumn{2}{|c|}{$3 / 10(30 \%)$} \\
\hline Vaccinated & $0 / 2(0 \%)$ & \\
\hline \multicolumn{3}{|c|}{30 day post-operative SARS Cov-2 positivity rate" } \\
\hline Unvaccinated & $4 / 9(44.4 \%)$ & \multirow{2}{*}{$p=0.0001$} \\
\hline Vaccinated & $0 / 32(0 \%)$ & \\
\hline Severe COVID-19 requiring hospitalisation & $2 / 4(50 \%)$ & \\
\hline $\begin{array}{l}\text { Post-operative day of positive RT/PCR and } \\
\text { symptomatic for COVIID-19 }\end{array}$ & $\begin{array}{l}\text { Mean - } 8.7 \\
\text { Median -9 }\end{array}$ & \\
\hline
\end{tabular}

Result(s)* In the overall cohort of 53 patients, 34 got vaccinated suggesting compliance of $64 \%$. In the unvaccinated cohort, $52.6 \%$ were p $\square$ e-surgery RT/PCR +ve against $5.8 \%$ vaccinated patients $(\mathrm{p}=0.0001)$. Thirty- day post-operative SARS Cov- 2 rate was $44.4 \%$ and $0 \%$ in the unvaccinated and vaccinated cohort respectively $(\mathrm{p}=0.0001)$. No cases of severe COVID-19 requiring hospitalisation were seen in the vaccinated cohort. There was no 30-day post-operative mortality in either cohorts.

Conclusion* Counselling regarding COVID-19 vaccination prior to surgery should be an essential part of pre-operative work up. COVID-19 vaccination prior to surgery has two-fold advantage. It prevents the postponement of elective cancer surgeries which are time bound. There is a significant decreased risk of severe COVID-19 infection and related morbidity post-operatively in the vaccinated population.

\section{SAME DAY DISCHARGE IN MINIMALLY INVASIVE SURGERY FOR GYNAECOLOGICAL CANCER}

C Hickish*, J Dilley, S Abdi, S Phadnis, E Brockbank. The Royal London Hospital, Gynaecology Oncology, LONDON, UK

\subsection{6/ijgc-2021-ESG0.300}

Introduction/Background* Same-day discharge (SDD) has been found to be safe and attainable following minimally invasive surgery (MIS) for gynaecological cancer. ${ }^{1}$ We audited the compliance to SDD, opposed to 24 hours discharge, at the Royal London Hospital.

Methodology We performed a retrospective audit of all minimally invasive hysterectomies performed at the Royal London Hospital in 2019 and 2020. All patients were identified for SDD at pre-op clinic and were followed up until 30 days post discharge.

Data was collected from electronic patient to record demographic, operating time \& outcome, postoperative recovery $(+/-$ complication and readmission) and time of discharge.

Following the first audit cycle in 2019, interventions were performed comprising of staff education to highlight the human factors that led to failed SDD (including delayed prescriptions, discharge summaries and removal of catheters).

Result(s)* A total of 12 patients were selected for SDD in 2020 compared to 22 in 2019 with 30 day follow up for all patients. Numbers of patient in 2020 was reduced due to covid. Successful SDD was achieved in $42 \%$ of cases in 2020 compared to $56 \%$ in 2019. Of those planned for SDD, $50 \%$ of those that failed were due to unavoidable intraoperative complications. No SDD discharges in 2020 failed due to pharmacy, transport or discharge documentation delays $(7$ failed SDD in 2019 due to these factors). No re-admissions or complications were recorded.

Conclusion* Same day discharge continues to be safe and achievable following minimally invasive surgery for gynecological cancer, despite disruption from the Covid-19 pandemic. Auditing and implementation of interventions helps improve this pathway.

\section{REFERENCE}

1. Korsholm M, Mogensen O, Jeppesen MM, Lysdal VK, Traen K, Jensen PT. Systematic review of same-day discharge after minimally invasive hysterectomy. Int J Gynaecol Obstet 2017 Feb;136(2):128-137. doi: 10.1002/ijgo.12023. Epub 2016 Nov 11. PMID: 28099736. 